\title{
GROWTH THEOREMS IN SLICE ANALYSIS OF SEVERAL VARIABLES
}

\author{
GUANGBIN REN, TING YANG
}

\begin{abstract}
In this paper, we define a class of slice mappings of several Clifford variables, and the corresponding slice regular mappings. Furthermore, we establish the growth theorem for slice regular starlike or convex mappings on the unit ball of several slice Clifford variables, as well as on the bounded slice domain which is slice starlike and slice circular.
\end{abstract}

\section{INTRODUCTION}

Ghiloni and Perotti [11] initiated the study of slice analysis on the quadratic cones of real alternative algebras; see [13, 19] for its recent development. The theory has some distinguished models on quaternions, real Clifford algebras, and Octonions; see [8, 9, [11, 12, 3], 2, 44, 10 for the pioneering works. It has important applications in the functional calculus for non-commutative operators $[5$.

Recently, the slice theory of several variables has also been studied [1], [6], [14, 21. In this article, we shall provide a new generalization, which enable us to extend slice analysis to higher dimensions as well as to the setting of several slice Clifford variables.

Based on a new convex combination identity in [18, the sharp growth theorems for slice monogenic extensions of univalent functions on the unit disc $D \subset \mathbb{C}$ in the setting of Clifford algebras was established as follows:

Let $f$ be a slice monogenic function on the unit ball $\mathbb{B}$ in the regular quadratic cone $\mathbb{R}^{(m+1)}$ (in this paper, $\mathbb{R}^{(m+1)}$ denotes $\mathbb{R}^{m+1}$ in [18) of $\mathbb{R}_{m}$ such that its restriction $f_{I}$ to $\mathbb{B}_{I}$ is injective and such that $f\left(\mathbb{B}_{I}\right) \subseteq \mathbb{C}_{I}$ for some $I \in \mathbb{S}_{m}$. If $f(0)=0, f^{\prime}(0)=1$, then

$$
\frac{|x|}{(1+|x|)^{2}} \leq|f(x)| \leq \frac{|x|}{(1-|x|)^{2}}, \quad x \in \mathbb{B} .
$$

Moreover, equality holds for one of these two inequalities at some point $x_{0} \in \mathbb{B} \backslash\{0\}$ if and only if $f$ is of the form $f(x)=x\left(1-x e^{I \theta}\right)^{-* 2}, \forall x \in \mathbb{B}$, for some $\theta \in \mathbb{R}$. (See 18] for details.)

From the classical geometric function theory in higher dimensions, it is known that the growth theorems fail for the full class of normalized univalent mappings [15]. It is Cartan who suggested to consider the subclass of starlike or convex mapping instead.

Date: October 26, 2018.

2010 Mathematics Subject Classification. Primary 32A22; Secondary 32A10, 32H02.

Key words and phrases. Clifford algebras; several complex variables; slice regular mappings; growth theorem.

The first author is supported by the NNSF of China (11771412). 
The aim of this paper is to generalize the growth results for subclasses of normalized univalent mappings on the unit ball in $\mathbb{C}^{n}$ to the subset of the several slice Clifford variables $\left(\mathcal{Q}^{m}\right)_{s}^{n}$.

Since the convex combination identity mentioned above was only proved for one-dimensional case, we overcome this problem by using a new approach. As an application, we obtain the growth theorems for slice regular starlike or convex mappings on the unit ball of several slice Clifford variables, as well as on a more general slice domain which is slice starlike and slice circular.

\section{Priliminaries}

The real Clifford algebra $\mathbb{R}_{m}$ is an universal associative algebra over $\mathbb{R}$ generated by $m$ basis elements $e_{1}, \cdots, e_{m}$, subject to the relations

$$
e_{\imath} e_{\jmath}+e_{\jmath} e_{\imath}=-2 \delta_{\imath \jmath}, \quad \imath, \jmath=1, \cdots, m .
$$

As a real vector space, $\mathbb{R}_{m}$ has dimension $2^{m}$. Each element $x$ in $\mathbb{R}_{m}$ can be expressed as

$$
x=\sum_{A \in \mathcal{P}(m)} x_{A} e_{A},
$$

where

$$
\mathcal{P}(m)=\left\{\left(h_{1}, \cdots, h_{r}\right) \in \mathbb{N}^{r} \mid r=1, \cdots, m, 1 \leq h_{1}<\cdots<h_{r} \leq m\right\} .
$$

For each $A=\left\{\left(h_{1}, \cdots, h_{r}\right) \in \mathcal{P}(m)\right\}$, the coefficients $x_{A} \in \mathbb{R}$, and the products $e_{A}:=e_{h_{1}} e_{h_{2}} \cdots e_{h_{r}}$ are the basis elements of the Clifford algebra $\mathbb{R}_{m}$. The unit of the Clifford algebra corresponds to $A=\varnothing$, and we set $e_{\phi}=1$. As usual, we identify the real numbers field $\mathbb{R}$ with the subalgebra of $\mathbb{R}_{m}$ generated by the unit.

Let $x^{c}$ be the Clifford conjugate of $x \in \mathbb{R}_{m}$. We define

$$
t(x)=x+x^{c}, \quad n(x)=x x^{c}
$$

to be the trace and the (squared) norm of a Clifford element $x$, respectively.

The quadratic cone of the Clifford algebra $\mathbb{R}_{m}[14$ is defined by

$$
\mathcal{Q}_{m}:=\mathbb{R} \cup\left\{x \in \mathbb{R}_{m} \mid t(x) \in \mathbb{R}, n(x) \in \mathbb{R}, 4 n(x)>t(x)^{2}\right\},
$$

and the space of paravectors is defined by

$$
\mathbb{R}^{(m+1)}:=\left\{x \in \mathbb{R}_{m} \mid[x]_{k}=0 \text { for every } k>1\right\},
$$

where $[x]_{k}$ denotes the k-vector pair of $x$.

We also set

$$
\mathbb{S}_{m}:=\left\{J \in \mathcal{Q}_{m} \mid J^{2}=-1\right\} .
$$

The elements of $\mathbb{S}_{m}$ are called the square roots of -1 in the Clilfford algebra $\mathbb{R}_{m}$. It is known that

$$
\mathbb{S}_{m}=\left\{x \in \mathbb{R}_{m} \mid t(x)=0, n(x)=1\right\} .
$$

We consider the cartesian product $\left(\mathbb{R}_{m}\right)^{n}$ of the Clifford algebra $\mathbb{R}_{m}$. Its complexification is denoted by

$$
\left(\mathbb{R}_{m}\right)_{\mathbb{C}}^{n}=\left(\mathbb{R}_{m}\right)^{n} \otimes_{\mathbb{R}} \mathbb{C}:=\left(\mathbb{R}_{m}\right)^{n}+i\left(\mathbb{R}_{m}\right)^{n} .
$$

For each $x, y \in \mathbb{R}^{n}$, we define

$$
\overline{x+i y}=x-i y
$$


be the complex conjugation of $x+i y$ in $\mathbb{R}_{\mathbb{C}}^{n}$. Note that $\mathbb{R}_{0}=\mathbb{R}$ and $\left(\mathbb{R}_{0}\right)_{\mathbb{C}}^{n}=\mathbb{C}^{n}$, then $\overline{x+i y}=x-i y$, for each $x, y \in \mathbb{R}^{n}$.

Definition 2.1. Let $D \subset \mathbb{C}^{n}$ be an open subset. A mapping $F: D \rightarrow\left(\mathbb{R}_{m}\right)_{\mathbb{C}}^{n}$ is called a $\mathbb{R}_{m}$-stem mapping over the domain $D$ if $F$ is complex intrinsic, i.e.

$$
F(\bar{z})=\overline{F(z)}, \quad \forall z \in D .
$$

Remark 2.2. (1) A mapping $F$ is a $\mathbb{R}_{m}$-stem mapping if and only if the $\mathbb{R}_{m}$-valued components $F_{1}, F_{2}$ of the $F=F_{1}+i F_{2}$ form an even-odd pair, i.e.

$$
F_{1}(\bar{z})=F_{1}(z), \quad F_{2}(\bar{z})=-F_{2}(z)
$$

for each $z \in D$.

(2)Consider $\mathbb{R}_{m}$ as a $2^{m}$-dimensional real vector space. By means of a basis $\mathcal{B}=\left\{e_{A}\right\}_{A \in \mathcal{P}_{m}}$ of $\mathbb{R}_{m}, F$ can be identity with complex intrinsic curves in $\mathbb{C}^{n}$.

Let $F(z)=F_{1}(z)+i F_{2}(z)=\sum_{A \in \mathcal{P}_{m}} F^{A} e_{A}$ with $F^{A}(z) \in \mathbb{C}^{n}$. Then

$$
\tilde{F}=\left(\begin{array}{cccc}
F_{1}^{0} & F_{1}^{1} & \cdots & F_{1}^{2^{m}-1} \\
F_{2}^{0} & F_{2}^{1} & \cdots & F_{2}^{2^{m}-1} \\
\vdots & \vdots & \ddots & \vdots \\
F_{n}^{0} & F_{n}^{1} & \cdots & F_{n}^{2^{m}}-1
\end{array}\right): D \rightarrow \mathbb{C}^{n \times 2^{m}}
$$

satisfies $\tilde{F}(\bar{z})=\overline{\tilde{F}(z)}$. Giving $\mathbb{R}_{m}$ the unique manifold structure as a real vector space, we get that a stem mapping $F$ is of class $C^{k}(k=0, \cdots, \infty)$ or real-analytic if and only if the same property for $\tilde{F}$. This notion is clearly independent of the choice of the basis of $\mathbb{R}_{m}$.

(3) In the case of $n=1$, Definition 2.1 was first introduced by Ghiloni and Petrotti [11].

In the several Clifford numbers $\left(\mathbb{R}_{m}\right)^{n}$, we define

$$
\left(\mathcal{Q}_{m}\right)_{s}^{n}:=\bigcup_{I \in \mathbb{S}_{m}} \mathbb{C}_{I}^{n}
$$

where

$$
\mathbb{C}_{I}^{n}:=\mathbb{R}^{n}+I \mathbb{R}^{n}, \quad I \in \mathbb{S}_{m}
$$

Let $\left(\mathbb{R}^{(m+1)}\right)^{n}$ be the space of multi-paravectors. Its subspace

$$
\left(\mathbb{R}^{(m+1)}\right)_{s}^{n}:=\left(\mathbb{R}^{(m+1)}\right)^{n} \bigcap\left(\mathcal{Q}_{m}\right)_{s}^{n}
$$

is called the space of slice multi-paravectors.

For any $\alpha, \beta \in \mathbb{R}^{n}, I \in \mathbb{S}_{m}$ with $z=\alpha+\beta I$, we set

$$
[z]:=\bigcup_{J \in \mathbb{S}_{m}} \alpha+\beta J \subseteq\left(\mathcal{Q}_{m}\right)_{s}^{n} .
$$

Given an open subset $D$ of $\mathbb{C}^{n}$, let $\Omega_{D}$ be the subset of $\left(\mathcal{Q}_{m}\right)_{s}^{n}$ obtained by the action on $D$ of the square roots of -1 :

$$
\Omega_{D}:=\left\{\alpha+\beta J \in\left(\mathcal{Q}_{m}\right)_{s}^{n} \mid \alpha+i \beta \in D, \alpha, \beta \in \mathbb{R}^{n}, J \in \mathbb{S}_{m}\right\},
$$

and

$$
D_{I}:=\Omega_{D} \cup \mathbb{C}_{I}^{n}, \quad I \in \mathbb{S}_{m},
$$


then

$$
\Omega_{D}=\bigcup_{I \in \mathbb{S}_{m}} D_{I}, \quad I \in \mathbb{S}_{m}
$$

In particular, $\Omega_{D}=\left(\mathcal{Q}_{m}\right)_{s}^{n}$ when $D=\mathbb{C}^{n}$.

Definition 2.3. Any stem mapping $F: D \rightarrow\left(\mathbb{R}_{m}\right)_{\mathbb{C}}^{n}$ induces a (left) slice mapping

$$
f=\mathcal{I}(F): \Omega_{D} \rightarrow\left(\mathbb{R}_{m}\right)^{n} .
$$

If $x=\alpha+I \beta \in D_{I}, \forall I \in \mathbb{S}_{m}$, we set

$$
f(x):=F_{1}(z)+I F_{2}(z) \quad(z=\alpha+i \beta) .
$$

The slice function $f$ is well defined, since $\left(F_{1}, F_{2}\right)$ is an even-odd pair w.r.t. $\beta$ and then $f(\alpha+(-\beta)(-J))=F_{1}(\bar{z})+(-J) F_{2}(\bar{z})=F_{1}(z)+J F_{2}(z)$. There is an analogous definition for right slice mappings when the element $J \in \mathbb{S}_{m}$ is replace on the right of $F_{2}(z)$. In what follows, the term slice mapping will always mean left slice mappings.

We will denote the set of all (left) slice mappings on $\Omega_{D}$ by

$$
\begin{aligned}
& \mathcal{S}\left(\Omega_{D},\left(\mathbb{R}_{m}\right)^{n}\right):=\left\{f: \Omega_{D} \rightarrow\left(\mathbb{R}_{m}\right)^{n} \mid f=\mathcal{I}(F),\right. \\
&\left.F: D \rightarrow\left(\mathbb{R}_{m}\right)_{\mathbb{C}}^{n} \text { is a } \mathbb{R}_{m} \text {-stem mapping }\right\} .
\end{aligned}
$$

The distinguished property for a slice mapping is its representation formula:

Proposition 2.4. Let $f \in \mathcal{S}\left(\Omega_{D},\left(\mathbb{R}_{m}\right)^{n}\right)$ and $J, K \in \mathbb{S}_{m}$ with $J \neq K$. Then

$$
f(\alpha+\beta I)=(I-K)\left((J-K)^{-1} f(\alpha+\beta J)\right)-(I-J)\left((J-K)^{-1} f(\alpha+\beta K)\right)
$$

for each $I \in \mathbb{S}_{m}, \alpha, \beta \in \mathbb{R}^{n}$ with $\alpha+I \beta \in D_{I}$.

Proof. For $f \in \mathcal{S}\left(\Omega_{D},\left(\mathbb{R}_{m}\right)^{n}\right)$, by definition,

$$
f(\alpha+\beta J)-f(\alpha-\beta K)=(J-K) F_{2}(\alpha+\beta i)
$$

Hence

$$
F_{2}(\alpha+\beta i)=(J-K)^{-1}(f(\alpha+\beta J)-f(\alpha+\beta K))
$$

and

$$
\begin{aligned}
F_{1}(\alpha+\beta i) & =f(\alpha+\beta J)-J F_{2}(\alpha+\beta i) \\
& =f(\alpha+\beta J)-J\left((J-K)^{-1}(f(\alpha+\beta J)-f(\alpha+\beta K))\right) .
\end{aligned}
$$

Therefore,

$$
\begin{aligned}
f(\alpha+\beta I) & =F_{1}(\alpha+\beta i)+I F_{2}(\alpha+\beta i) \\
& =f(\alpha+\beta J)+(I-J)\left((J-K)^{-1}(f(\alpha+\beta J)-f(\alpha+\beta K))\right) \\
& =(J-K+I-J)\left((J-K)^{-1} f(\alpha+\beta J)\right)+(I-J)\left((J-K)^{-1} f(\alpha+\beta K)\right) \\
& =(I-K)\left((J-K)^{-1} f(\alpha+\beta J)\right)+(I-J)\left((J-K)^{-1} f(\alpha+\beta K)\right) .
\end{aligned}
$$

By settting $K=-J$ in Proposition 2.4 we obtain the following corollary.

Corollary 2.5. Let $f \in \mathcal{S}\left(\Omega_{D},\left(\mathbb{R}_{m}\right)^{n}\right)$ and $I \in \mathbb{S}_{m}, \alpha, \beta \in \mathbb{R}^{n}$ with $\alpha+I \beta \in \Omega_{D}$. Then

$$
f(\alpha+\beta I)=\frac{1}{2}(f(\alpha+\beta J)+f(\alpha-\beta J))-\frac{I}{2}(J(f(\alpha+\beta J)-f(\alpha-\beta J)))
$$

for each $J \in \mathbb{S}_{m}$. 
We will denote by

$$
\mathcal{S}^{1}\left(\Omega_{D},\left(\mathbb{R}_{m}\right)^{n}\right):=\left\{f=\mathcal{I}(F) \in \mathcal{S}\left(\Omega_{D},\left(\mathbb{R}_{m}\right)^{n}\right) \mid F \in C^{1}\left(D,\left(\mathbb{R}_{m}\right)_{\mathbb{C}}^{n}\right)\right\}
$$

the real vector space of slice mappings of several Clifford variables with stem mapping of class $C^{1}$.

Let $f=\mathcal{I}(F) \in \mathcal{S}^{1}\left(\Omega_{D},\left(\mathbb{R}_{m}\right)^{n}\right)$ and $z=\alpha+i \beta \in D$. Then the partial derivatives $\partial F / \partial \alpha_{t}$ and $i \partial F / \partial \beta_{t}$ are continous $\mathbb{R}_{m}-$ stem mappings on $D$, for $t=1,2, \cdots, n$. The same property holds for their linear combinations

$$
\frac{\partial F}{\partial z_{t}}=\frac{1}{2}\left(\frac{\partial F}{\partial \alpha_{t}}-i \frac{\partial F}{\partial \beta_{t}}\right) \quad \text { and } \quad \frac{\partial F}{\partial \bar{z}_{t}}=\frac{1}{2}\left(\frac{\partial F}{\partial \alpha_{t}}+i \frac{\partial F}{\partial \beta_{t}}\right)
$$

where $z=\left(z_{1}, \cdots, z_{n}\right), \alpha=\left(\alpha_{1}, \cdots, \alpha_{n}\right), \beta=\left(\beta_{1}, \cdots, \beta_{n}\right), t=1,2, \cdots, n$.

Definition 2.6. Let $f=\mathcal{I}(F) \in \mathcal{S}^{1}\left(\Omega_{D},\left(\mathbb{R}_{m}\right)^{n}\right)$. We set

$$
\frac{\partial f}{\partial x}:=\mathcal{I}\left(\frac{\partial F}{\partial z}\right), \quad \text { and } \quad \frac{\partial f}{\partial \bar{x}}:=\mathcal{I}\left(\frac{\partial F}{\partial \bar{z}}\right),
$$

i.e.

$$
\left(\frac{\partial f}{\partial x_{1}}, \cdots, \frac{\partial f}{\partial x_{n}}\right)=\left(\mathcal{I}\left(\frac{\partial F}{\partial z_{1}}\right), \cdots, \mathcal{I}\left(\frac{\partial F}{\partial z_{n}}\right)\right)
$$

and

$$
\left(\frac{\partial f}{\partial \bar{x}_{1}}, \cdots, \frac{\partial f}{\partial \bar{x}_{n}}\right)=\left(\mathcal{I}\left(\frac{\partial F}{\partial \bar{z}_{1}}\right), \cdots, \mathcal{I}\left(\frac{\partial F}{\partial \bar{z}_{n}}\right)\right)
$$

where

$$
\mathcal{I}\left(\frac{\partial F}{\partial z_{t}}\right)=\left(\mathcal{I}\left(\frac{\partial F^{1}}{\partial z_{t}}\right), \mathcal{I}\left(\frac{\partial F^{2}}{\partial z_{t}}\right), \cdots, \mathcal{I}\left(\frac{\partial F^{n}}{\partial z_{t}}\right)\right)^{T}
$$

and

$$
\mathcal{I}\left(\frac{\partial F}{\partial \bar{z}_{t}}\right)=\left(\mathcal{I}\left(\frac{\partial F^{1}}{\partial \bar{z}_{t}}\right), \mathcal{I}\left(\frac{\partial F^{2}}{\partial \bar{z}_{t}}\right), \cdots, \mathcal{I}\left(\frac{\partial F^{n}}{\partial \bar{z}_{t}}\right)\right)^{T},
$$

for $F=\left(F^{1}, F^{2}, \cdots, F^{n}\right), t=1,2, \cdots, n$, and $A^{T}$ stands for the transpose of the vector $A$.

The notation $\partial f / \partial \bar{x}_{t}$ is justified by the following properties:

$$
\bar{x}=\left(\mathcal{I}\left(\bar{z}_{1}\right), \cdots, \mathcal{I}\left(\bar{z}_{n}\right)\right)^{T}
$$

and therefore

$$
\partial \bar{x} / \partial \bar{x}_{t}=(0, \cdots, 0,1,0, \cdots, 0)^{T}, \quad \partial x / \partial \bar{x}_{t}=0 .
$$

Left multiplication by $i$ defines a complex structure on $\left(\mathbb{R}_{m}\right)_{\mathbb{C}}^{n}$. With respect to this structure, a $C^{1}$ mapping $F=F_{1}+i F_{2}: D \rightarrow\left(\mathbb{R}_{m}\right)_{\mathbb{C}}^{n}$ is holomorphic if and only if its components $F_{1}, F_{2}$ satisfy the Cauchy-Riemann equations:

$$
\frac{\partial F_{1}}{\partial \alpha}=\frac{\partial F_{2}}{\partial \beta}, \quad \frac{\partial F_{1}}{\partial \beta}=-\frac{\partial F_{2}}{\partial \alpha},
$$

i.e.

$$
\frac{\partial F}{\partial \bar{z}}=0, \quad(z=\alpha+i \beta \in D)
$$


where

and

$$
\frac{\partial F_{p}}{\partial \alpha}=\left(\begin{array}{cccc}
\frac{\partial F_{p}^{1}}{\partial \alpha_{1}} & \frac{\partial F_{p}^{1}}{\partial \alpha_{2}} & \cdots & \frac{\partial F_{p}^{1}}{\partial \alpha_{n}} \\
\frac{\partial F_{p}^{2}}{\partial \alpha_{1}} & \frac{\partial F_{p}^{2}}{\partial \alpha_{2}} & \cdots & \frac{\partial F_{p}^{2}}{\partial \alpha_{n}} \\
\vdots & \vdots & \ddots & \vdots \\
\frac{\partial F_{p}^{n}}{\partial \alpha_{1}} & \frac{\partial F_{p}^{n}}{\partial \alpha_{2}} & \cdots & \frac{\partial F_{p}^{n}}{\partial \alpha_{n}}
\end{array}\right)
$$

$$
\frac{\partial F_{p}}{\partial \beta}=\left(\begin{array}{cccc}
\frac{\partial F_{p}^{1}}{\partial \beta_{1}} & \frac{\partial F_{p}^{1}}{\partial \beta_{2}} & \cdots & \frac{\partial F_{p}^{1}}{\partial \beta_{n}} \\
\frac{\partial F_{p}^{2}}{\partial \beta_{1}} & \frac{\partial F_{p}^{2}}{\partial \beta_{2}} & \cdots & \frac{\partial F_{p}^{2}}{\partial \beta_{n}} \\
\vdots & \vdots & \ddots & \vdots \\
\frac{\partial F_{p}^{n}}{\partial \beta_{1}} & \frac{\partial F_{p}^{n}}{\partial \beta_{2}} & \cdots & \frac{\partial F_{p}^{n}}{\partial \beta_{n}}
\end{array}\right)
$$

for $F_{p}=\left(F_{p}^{1}, \cdots, F_{p}^{n}\right), p=1,2$, and $\alpha=\left(\alpha_{1}, \cdots, \alpha_{n}\right), \beta=\left(\beta_{1}, \cdots, \beta_{n}\right)$.

This condition is equivalent to require that, for any basis $\mathcal{B}$, the mapping $\tilde{F}$ (cf. Remark 2.2) is holomorphic.

Set

$$
\frac{\partial f}{\partial x}:=\left(\begin{array}{cccc}
\frac{\partial f_{1}}{\partial x_{1}} & \frac{\partial f_{1}}{\partial x_{2}} & \cdots & \frac{\partial f_{1}}{\partial x_{n}} \\
\frac{\partial f_{2}}{\partial x_{1}} & \frac{\partial f_{2}}{\partial x_{2}} & \cdots & \frac{\partial f_{2}}{\partial x_{n}} \\
\vdots & \vdots & \ddots & \vdots \\
\frac{\partial f_{n}}{\partial x_{1}} & \frac{\partial f_{n}}{\partial x_{2}} & \cdots & \frac{\partial f_{n}}{\partial x_{n}}
\end{array}\right), \quad \frac{\partial F}{\partial z}:=\left(\begin{array}{cccc}
\frac{\partial F^{1}}{\partial z_{1}} & \frac{\partial F^{1}}{\partial z_{2}} & \cdots & \frac{\partial F^{1}}{\partial z_{n}} \\
\frac{\partial F^{2}}{\partial z_{1}} & \frac{\partial F^{2}}{\partial z_{2}} & \cdots & \frac{\partial F^{2}}{\partial z_{n}} \\
\vdots & \vdots & \ddots & \vdots \\
\frac{\partial F^{n}}{\partial z_{1}} & \frac{\partial F^{n}}{\partial z_{2}} & \cdots & \frac{\partial F^{n}}{\partial z_{n}}
\end{array}\right) .
$$

Similarly define $\frac{\partial f}{\partial \bar{x}}$ and $\frac{\partial F}{\partial \bar{z}}$. Let

$$
\mathcal{H}(D):=\left\{F \in C^{1}\left(D,\left(\mathbb{R}_{m}\right)_{\mathbb{C}}^{n}\right): \frac{\partial F}{\partial \bar{z}}=0, \forall z=\left(z_{1}, \cdots, z_{n}\right) \in D\right\} .
$$

Definition 2.7. A (left) slice mapping $f=\mathcal{I}(F) \in \mathcal{S}^{1}\left(\Omega_{D},\left(\mathbb{R}_{m}\right)^{n}\right)$ is (left) slice regular if its stem mapping $F$ is holomorphic.

We denote the vector space of slice regular functions on $\Omega_{D}$ by $\mathcal{S R}\left(\Omega_{D},\left(\mathbb{R}_{m}\right)^{n}\right)$. That is,

$$
\mathcal{S R}\left(\Omega_{D},\left(\mathbb{R}_{m}\right)^{n}\right):=\left\{\mathcal{I}(F) \in \mathcal{S}^{1}\left(\Omega_{D},\left(\mathbb{R}_{m}\right)^{n}\right) \mid F: D \rightarrow\left(\mathbb{R}_{m}\right)_{\mathbb{C}}^{n} \text { is holomorphic }\right\} .
$$

Remark 2.8. If we define

$$
\mathbb{S}_{m}^{*}:=\left\{J \in \mathbb{R}^{(m+1)} \mid J^{2}=1\right\}
$$

and

$$
\Omega_{D}^{*}:=\left\{x=\alpha+\beta J \in\left(\mathbb{R}^{(m+1)}\right)_{s}^{n} \mid \alpha+i \beta \in D, \alpha, \beta \in \mathbb{R}^{n}, J \in \mathbb{S}_{m}^{*}\right\},
$$

then $\Omega_{D}^{*} \subseteq \Omega_{D}$ and $f \in \mathcal{S R}\left(\Omega_{D}^{*},\left(\mathbb{R}_{m}\right)^{n}\right)$ is called the slice monogenic mapping.

Remark 2.9. In the case of $m=2$, we call $f \in \mathcal{S R}\left(\Omega_{D}, \mathbb{H}^{n}\right)$ a slice regular mapping of several quaternionic variables.

Proposition 2.10. Let $f=\mathcal{I}(F) \in \mathcal{S}^{1}\left(\Omega_{D},\left(\mathbb{R}_{m}\right)^{n}\right)$. Then $f$ is slice regular on $\Omega_{D}$ if and only if the restriction

$$
f_{I}: D_{I} \rightarrow\left(\mathbb{R}_{m}\right)^{n}
$$

is holomorphic for every $I \in \mathbb{S}_{m}$. 
Proof. Notice that

$$
f_{I}(\alpha+I \beta)=F_{1}(\alpha+i \beta)+I F_{2}(\alpha+i \beta) .
$$

If $F$ is holomorphic then

$$
\frac{\partial f_{I}}{\partial \alpha}+I \frac{\partial f_{I}}{\partial \beta}=\frac{\partial F_{1}}{\partial \alpha}+I \frac{\partial F_{2}}{\partial \alpha}+I\left(\frac{\partial F_{1}}{\partial \beta}+I \frac{\partial F_{2}}{\partial \beta}\right)=0
$$

at every point $x=\alpha+I \beta \in D_{I}$.

Conversely, assume that $f_{I}$ is holomorphic at every $I \in \mathbb{S}_{m}$. Then

$$
0=\frac{\partial f_{I}}{\partial \alpha}+I \frac{\partial f_{I}}{\partial \beta}=\frac{\partial F_{1}}{\partial \alpha}-\frac{\partial F_{2}}{\partial \beta}+I\left(\frac{\partial F_{2}}{\partial \alpha}+\frac{\partial F_{1}}{\partial \beta}\right)
$$

at every point $z=\alpha+i \beta \in D$. From the arbitrariness of $I$ it follows that $F_{1}, F_{2}$ satisfy the Cauchy-Riemann equations.

Proposition 2.11. Let $f \in \mathcal{S R}\left(\Omega_{D},\left(\mathbb{R}_{m}\right)^{n}\right)$. For every choice of $I=I_{1} \in \mathbb{S}_{m}$, let $I_{2}, \cdots, I_{m}$ be a completion to an orthonormal basis of the algebra $\mathbb{R}_{m}$. Then there exists $2^{m-1}$ holomorphic mappings $F_{A}: D_{I} \rightarrow \mathbb{C}_{I}^{n}, A \in \mathcal{P}(m)$ such that for every $z=\alpha+I \beta \in D_{I}$ we have

$$
f_{I}(z)=\sum_{A \in \mathcal{P}(m)} F_{A}(z) I_{A}
$$

Here $I_{\varnothing}=1$.

\section{Growth theorems in the Unit Ball}

We consider the unit ball in the set of slice several Clifford variables $\left(\mathcal{Q}_{m}\right)_{s}^{n}$, i.e.

$$
\mathbb{B}:=\left\{x \in\left(\mathcal{Q}_{m}\right)_{s}^{n} \mid\|x\|=\left(\sum_{t=1}^{n}\left|x_{t}\right|^{2}\right)^{\frac{1}{2}}<1, x=\left(x_{1}, \cdots, x_{n}\right)\right\},
$$

then

$$
\mathbb{B}_{I}=\mathbb{B} \cap \mathbb{C}_{I}^{n}, \quad \forall I \in \mathbb{S}_{m} .
$$

Lemma 3.1. Let $f \in \mathcal{S}\left(\Omega_{D},\left(\mathbb{R}_{m}\right)^{n}\right), f\left(D_{I}\right) \subseteq \mathbb{C}_{I}^{n}$ for some $I \in \mathbb{S}_{m}$. Then

$$
\begin{aligned}
& \max _{J \in \mathbb{S}_{m}}\|f(\alpha+J \beta)\|=\max _{J= \pm I}\|f(\alpha+J \beta)\|, \\
& \min _{J \in \mathbb{S}_{m}} \| f\left((\alpha+J \beta)\left\|=\min _{J= \pm I}\right\| f(\alpha+J \beta) \|,\right.
\end{aligned}
$$

for each $\alpha, \beta \in \mathbb{R}^{n}, J \in \mathbb{S}_{m}$ with $\alpha+J \beta \in \Omega_{D}$.

Proof. Since $f \in \mathcal{S}\left(\Omega_{D},\left(\mathbb{R}_{m}\right)^{n}\right)$, the representation formula shows that

$$
f(x)=\frac{1}{2}(f(z)+f(\bar{z}))-J \frac{I}{2}(f(z)-f(\bar{z}))
$$

for each $u, v \in \mathbb{R}^{m}$ and $J \in \mathbb{S}_{m}$ with $x=u+J v \in D_{J}$ and $z=u+I v$.

Denote

$$
\begin{aligned}
\alpha & =\frac{1}{2}(f(z)+f(\bar{z})), \\
\beta & =-\frac{I}{2}(f(z)-f(\bar{z})) .
\end{aligned}
$$

By assumption, we have both $\alpha$ and $\beta$ in $\mathbb{C}_{I}^{n}$. We set

$$
\alpha=\left(\alpha_{1}, \cdots, \alpha_{n}\right), \quad \beta=\left(\beta_{1}, \cdots, \beta_{n}\right) .
$$


For $\alpha_{t} \neq 0$, we set

$$
\beta_{t} \alpha_{t}^{-1}=a_{t}+I b_{t}
$$

where $a_{t}, b_{t} \in \mathbb{R}$ for any $t \in\{1, \cdots, n\}$.

Take $I_{2}, \cdots, I_{2^{m}-1} \in \mathbb{S}_{m}$ such that $\left\{1, I, I_{2}, \cdots, I_{2^{m}-1}\right\}$ consists of a basis of $\mathbb{R}_{m}$. For any $J \in \mathbb{S}_{m}$ we can represent it under such a basis as

$$
J=u I+\sum_{l=2}^{2^{m}-1} v_{l} I_{l}
$$

with real coefficients $u, v_{2}, v_{3}, \cdots, v_{2^{m}-1} \in \mathbb{R}$ such that

$$
u^{2}+\sum_{l=2}^{2^{m}-1} v_{l}^{2}=1 \text {. }
$$

We can rewrite

$$
f(x)=\alpha+J \beta,
$$

then

$$
\begin{aligned}
\|f(x)\|^{2} & =\sum_{t=1}^{n}\left|\alpha_{t}+J \beta_{t}\right|^{2} \\
& =\sum_{t=1, \alpha_{t} \neq 0}^{n}\left|1+J \beta_{t} \alpha_{t}^{-1}\right|^{2}\left|\alpha_{t}\right|^{2}+\sum_{t=1, \alpha_{t}=0}^{n}\left|\beta_{t}\right|^{2} \\
& =\sum_{t=1, \alpha_{t} \neq 0}^{n}\left|1+\left(u I+\sum_{l=2}^{2^{m}-1} v_{l} I_{l}\right)\left(a_{t}+I b_{t}\right)\right|^{2}\left|\alpha_{t}\right|^{2}+\sum_{t=1, \alpha_{t}=0}^{n}\left|\beta_{t}\right|^{2} \\
& =\sum_{t=1, \alpha_{t} \neq 0}^{n}\left(1+a_{t}^{2}+b_{t}^{2}-2 b_{t} u\right)\left|\alpha_{t}\right|^{2}+\sum_{t=1, \alpha_{t}=0}^{n}\left|\beta_{t}\right|^{2} \\
& =\sum_{t=1, \alpha_{t} \neq 0}^{n}\left(1+a_{t}^{2}+b_{t}^{2}\right)\left|\alpha_{t}\right|^{2}+\sum_{t=1, \alpha_{t}=0}^{n}\left|\beta_{t}\right|^{2}-\sum_{t=1}^{n} 2 b_{t}\left|\alpha_{t}\right|^{2} u \\
& =: g(u)
\end{aligned}
$$

Therefore

$$
\begin{aligned}
& \|f(x)\|_{\max }=\max _{u \in[-1,1]} g(u)=\max _{u= \pm 1} g(u), \\
& \|f(x)\|_{\min }=\min _{u \in[-1,1]} g(u)=\min _{u= \pm 1} g(u) .
\end{aligned}
$$

Remark 3.2. In [18], it obtained the similar result to Lemma 3.1 in the case of $n=1$. The method above is simpler and satisfies in higher dimensions. Moreover, $f$ does not need to be a slice monogenic mapping on $\Omega_{D}^{*}$ (see Remark 2.8), it only need to be a slice mapping on $\Omega_{D}$.

Theorem 3.3. Let $f$ be a mapping in $\mathcal{S R}\left(\mathbb{B},\left(\mathbb{R}_{m}\right)^{n}\right)$ such that its restriction $f_{I}$ to $\mathbb{B}_{I}$ is a starlike mapping such that $f\left(\mathbb{B}_{I}\right) \subseteq \mathbb{C}_{I}^{n}$ for some $I \in \mathbb{S}_{m}$. If $f(0)=$ $0, f^{\prime}(0)=\mathbb{I}_{n}$ (Identity matrix of order $n$ ), then

$$
\frac{\|x\|}{(1+\|x\|)^{2}} \leq\|f(x)\| \leq \frac{\|x\|}{(1-\|x\|)^{2}}, \quad \forall x \in \mathbb{B} .
$$

These estimates are sharp. 
Proof. Since $f_{I}: \mathbb{B}_{I} \subset \mathbb{C}_{I}^{n} \rightarrow \mathbb{C}_{I}^{n}$ is a starlike mapping such that $f_{I}(0)=0, f_{I}^{\prime}(0)=$ $\mathbb{I}_{n}$, we have 15

$$
\frac{\|z\|}{(1+\|z\|)^{2}} \leq\left\|f_{I}(z)\right\| \leq \frac{\|z\|}{(1-\|z\|)^{2}} \quad \forall z \in \mathbb{B}_{I} .
$$

With $z$ replaced by $\bar{z}$, then

$$
\frac{\|z\|}{(1+\|z\|)^{2}} \leq\left\|f_{I}(\bar{z})\right\| \leq \frac{\|z\|}{(1-\|z\|)^{2}} \quad \forall \bar{z} \in \mathbb{B}_{I} .
$$

These estimates are sharp.

For any $x \in \mathbb{B}$, there exists $z=\alpha+I \beta \in \mathbb{B}_{I}$ and $J \in \mathbb{S}_{m}$ such that $x=\alpha+J \beta \in \mathbb{B}$ Lemma 3.1 concludes that

$$
\|f(x)\| \geq \min \left\{\left\|f_{I}(z)\right\|,\left\|f_{I}(\bar{z})\right\|\right\} \geq \frac{\|z\|}{(1+\|z\|)^{2}}=\frac{\|x\|}{(1+\|x\|)^{2}} .
$$

This estimate is sharp.

The reverse inequality can be proved similarly.

Example 3.4. We consider the function Let $f: \mathbb{B} \rightarrow\left(\mathbb{R}_{m}\right)^{n}$, defined by

$$
f(x)=\left(x_{1}\left(1-x_{1} e^{I \theta}\right)^{-* 2}, \cdots, x_{n}\left(1-x_{n} e^{I \theta}\right)^{-* 2}\right) .
$$

It is easy to see that $f \in \mathcal{S R}\left(\mathbb{B},\left(\mathbb{R}_{m}\right)^{n}\right), f\left(\mathbb{B}_{I}\right) \subseteq \mathbb{C}_{I}^{n}$ and $f(0)=0, f^{\prime}(0)=I_{n}$. Since $f_{I}$ is a starlike mapping on $\mathbb{B}_{I}$ (see [15]), we have $f$ satisfies all the conditions of Theorem 3.3 .

For convex mappings, the same approach yields the similar results.

Theorem 3.5. Let $f$ be a mapping in $\mathcal{S R}\left(\mathbb{B},\left(\mathbb{R}_{m}\right)^{n}\right)$ such that its restriction $f_{I}$ to $\mathbb{B}_{I}$ is a convex mapping such that $f\left(\mathbb{B}_{I}\right) \subseteq \mathbb{C}_{I}^{n}$ for some $I \in \mathbb{S}_{m}$. If $f(0)=0, f^{\prime}(0)=$ $\mathbb{I}_{n}$, then

$$
\frac{\|x\|}{1+\|x\|} \leq\|f(x)\| \leq \frac{\|x\|}{1-\|x\|}, \quad \forall x \in \mathbb{B} .
$$

These estimates are sharp.

Theorem 3.6. Let $f \in \mathcal{S R}\left(\mathbb{B},\left(\mathbb{R}_{m}\right)^{n}\right)$ such that its restriction $f_{I}$ to $\mathbb{B}_{I}$ is a convex mapping such that $f\left(\mathbb{B}_{I}\right) \subseteq \mathbb{C}_{I}^{n}$ for some $I \in \mathbb{S}_{m}$. If $f(0)=0, f^{\prime}(0)=\mathbb{I}_{n}$ (Identity matrix of order $n$ ), then

$$
\frac{\|x\|}{1+\|x\|} \leq\|f(x)\| \leq \frac{\|x\|}{1-\|x\|} \quad x \in \mathbb{B} .
$$

These estimates are sharp.

Example 3.7. The function $f: \mathbb{B} \rightarrow\left(\mathbb{R}_{m}\right)^{n}$, defined by

$$
f(x)=\left(x_{1}\left(1-x_{1} e^{I \theta}\right), \cdots, x_{n}\left(1-x_{n} e^{I \theta}\right)\right),
$$

provides an example satisfying all the conditions of Theorem 3.6 . 


\section{Growth ThEOREMS IN THE STARLIKE OR CONVEX DOMAINS}

We shall now generalize the results in the last section to more general domains, other than the unit ball.

We consider the bounded slice domains which are slice starlike and slice circular.

Definition 4.1. A set $\Omega \in\left(\mathcal{Q}_{m}\right)_{s}^{n}$ is called starlike with respect to a fixed point $\omega_{0} \in \Omega$ if the closed line segment joining $\omega_{0}$ to each point $\omega \in \Omega$ lies entirely in $\Omega$. Also we say that $\Omega$ is convex if for all $\omega_{1}, \omega_{2} \in \Omega$, the closed line segment between $\omega_{1}$ and $\omega_{2}$ lies entirely in $\Omega$. In other words, $\Omega$ is convex if and only if $\Omega$ is starlike with respect to each of its points. The term starlike will mean starlike with respect to zero.

Definition 4.2. A set $\Omega \in\left(\mathcal{Q}_{m}\right)_{s}^{n}$ is called slice starlike if $\Omega_{I}$ is starlike in $\mathbb{C}_{I}^{n}$ for some $I \in \mathbb{S}_{m}$.

Definition 4.3. A domain $\Omega \in\left(\mathcal{Q}_{m}\right)_{s}^{n}$ is called slice circular, if for any $z \in$ $\Omega_{I}, \theta \in \mathbb{R}$, and $I, J \in \mathbb{S}_{m}$, we have $e^{J \theta} x \in \Omega$ for any $x \in[z] \cap \mathbb{C}_{J}^{n}$.

Remark 4.4. A domain $\Omega$ is slice circular if and only if for some $I \in \mathbb{S}_{m}$,

(1) $\Omega_{I}$ is circular (i.e. $e^{I \theta} z \in \Omega_{I}$ if $z \in \Omega_{I}$ and $\theta \in \mathbb{R}$ );

(2) $\Omega$ is axially symmetric (i.e. $x \in \Omega$ if $x \in[z]$ and $z \in \Omega_{I}$ ).

Obviously, the unit ball $\mathbb{B}$ and polydisc $\mathbb{P}$ are slice circular, where

$$
\mathbb{P}:=\left\{x \in\left(\mathcal{Q}_{m}\right)_{s}^{n}|| x_{t} \mid<1, x=\left(x_{1}, \cdots, x_{n}\right), t=1,2, \cdots, n\right\} .
$$

Definition 4.5. A domain $\Omega \in\left(\mathcal{Q}_{m}\right)_{s}^{n}$ is called slice domain, if

(1) $\Omega \cap \mathbb{R}^{n} \neq \emptyset$,

(2) $\Omega_{I}$ is a domain of $\left(\mathcal{Q}_{m}\right)_{s}^{n} \cap \mathbb{C}_{I}^{n}$, for any $I \in \mathbb{S}_{m}$.

The bounded slice starlike slice circular and slice domain has an analytic characterization via definition functions.

Lemma 4.6. An axially symmetric slice domain $\Omega \subseteq\left(\mathcal{Q}_{m}\right)_{s}^{n}$ is bounded slice starlike and slice circular if and only if there exists a unique continuous function

$$
\rho:\left(\mathcal{Q}_{m}\right)_{s}^{n} \rightarrow \mathbb{R}
$$

called the defining function of $\Omega$, such that

(1) $\rho(x) \geq 0, \forall x \in\left(\mathcal{Q}_{m}\right)_{s}^{n} ; \rho(x)=0 \Longleftrightarrow x=0$;

(2) $\rho(t x)=|t| \rho(x), \forall J \in \mathbb{S}_{m}, \forall x \in \mathbb{C}_{J}^{n}, t \in \mathbb{C}_{J}$, ;

(3) $\Omega=\left\{x \in\left(\mathcal{Q}_{m}\right)_{s}^{n}: \rho(x)<1\right\}$.

Proof. The proof is similar as the case of several complex variables [16]. If the continuous function $\rho(x)$ satisfies (1), (2), and (3), then clearly $\Omega$ is a starlike slice circular domain. Its boundedness is also easy to prove, since if there exists a ray coming from the origin which completely falls in the starlike domain $\Omega$, then for any fixed point $x_{0}$ in this ray we have from (3)

$$
\rho\left(t x_{0}\right)<1, \quad \forall t \in[0, \infty) .
$$

But we then obtain $\rho\left(x_{0}\right)=0$ in terms of $(2)$. This contradicts (1).

Conversely, if $\Omega$ is a bounded slice starlike and slice circular domain in $\left(\mathbb{R}^{m+1}\right)_{s}^{n}$, then we define

$$
\rho(x)=\inf \left\{c>0: c^{-1} x \in \Omega\right\}
$$


Obviously, $\rho(x)$ satisfies (2), (3), and $\rho(x) \geq 0$ for all $x$. If there exists a point $x_{0} \neq 0$, such that $\rho(x)=0$, then it follows from (2) and (3) that $\Omega$ includes the whole ray which comes from the origin and through the point $x_{0}$, hence $\Omega$ is unbounded. Thus (1) holds. Finally we prove that $\rho(x)$ is continuous. Clearly,

$$
\left\{x \in\left(\mathcal{Q}_{m}\right)_{s}^{n}: r<\rho(x)<R\right\}=R \Omega \backslash \overline{r \Omega}
$$

is an open set in $\left(\mathcal{Q}_{m}\right)_{s}^{n}$, which implies the continuity of $\rho$.

Remark 4.7. From the proof of Lemma 4.6, we have that

$$
\rho(x)=\rho(z), \quad \forall z \in[x] \subseteq \Omega,
$$

where $\Omega$ is a bounded slice starlike and slice circular and slice domain.

Now we can state our main result about the growth theorem in bounded starlike slice circular and slice domain.

Theorem 4.8. Let $\Omega_{D}$ be a bounded slice starlike slice circular slice domain in $\left(\mathcal{Q}_{m}\right)_{s}^{n}$, its defining function $\rho(x)$ is a $C^{1}$ function on $\Omega_{D}$ except for a lower dimensional set. If $f \in \mathcal{S R}\left(\Omega_{D},\left(\mathbb{R}_{m}\right)^{n}\right)$ such that its restriction $f_{I}$ to $D_{I}$ is a starlike mapping such that $f\left(D_{I}\right) \subseteq \mathbb{C}_{I}^{n}$. If $f(0)=0, f^{\prime}(0)=\mathbb{I}_{n}$ (Identity matrix of order $n)$, then for any $x \in \Omega_{D}$,

$$
\frac{\rho(x)}{(1+\rho(x))^{2}} \leq\|f(x)\| \leq \frac{\rho(x)}{(1-\rho(x))^{2}}
$$

or equivalently,

$$
\frac{\|x\|}{(1+\rho(x))^{2}} \leq\|f(x)\| \leq \frac{\|x\|}{(1-\rho(x))^{2}} .
$$

These estimates are sharp.

Proof. Since $\Omega_{D}$ be a bounded slice starlike slice circular domain in $\left(\mathcal{Q}_{m}\right)_{s}^{n}, D_{I}$ is bounded, complex circular in $\mathbb{C}_{I}^{n}$, and is also starlike in $\mathbb{C}_{I}^{n}$, for any $I \in \mathbb{S}_{m}$. By the assumption that $\rho(x)$ is a $C^{1}$ function on $\Omega_{D}$, we know that $\rho(z)$ is a $C^{1}$ function on $\mathbb{C}_{I}^{n}$. Notice that $f_{I}$ is a biholomorphic starlike mapping on $D_{I}$ and admits the properties that $f\left(D_{I}\right) \subseteq \mathbb{C}_{I}^{n}$ and $f(0)=0, f^{\prime}(0)=\mathbb{I}_{n}$. Thanks to the classical results in several complex variables [16], we have

$$
\frac{\rho(z)}{(1+\rho(z))^{2}} \leq\|f(z)\| \leq \frac{\rho(z)}{(1-\rho(z))^{2}}, \quad \forall z \in D_{I},
$$

as well as

$$
\frac{\|z\|}{(1+\rho(z))^{2}} \leq\|f(z)\| \leq \frac{\|z\|}{(1-\rho(z))^{2}} \quad \forall z \in D_{I} .
$$

By symmetrically, we also have

$$
\frac{\rho(\bar{z})}{(1+\rho(\bar{z}))^{2}} \leq\|f(\bar{z})\| \leq \frac{\rho(\bar{z})}{(1-\rho(\bar{z}))^{2}}, \quad \forall z \in D_{I} .
$$

These estimates are sharp.

Suppose $x=\alpha+J \beta \in \Omega_{D}$ for any $J \in \mathbb{S}_{m}$ and $z=\alpha+I \beta \in D_{I}$. By Lemma 3.1, we have

$$
\|f(x)\| \geq \min \left\{\left\|f_{I}(z)\right\|,\left\|f_{I}(\bar{z})\right\|\right\} \geq \frac{\rho(z)}{(1+\rho(z))^{2}}=\frac{\rho(x)}{(1+\rho(x))^{2}} .
$$

These estimates are sharp. The remaining inequality can be proved similarly. 
Likewise, we can establish the growth theorem in bounded convex slice circular domain for convex mappings.

Notice that since the convex domain is a starlike domain, for a bounded convex slice circular and slice domain $\Omega_{D}$, there also exists a definition function of the domain $\Omega_{D}$.

Theorem 4.9. Let $\Omega_{D}$ be a bounded convex slice circular slice domain in $\left(\mathcal{Q}_{m}\right)_{s}^{n}$, with defining function $\rho(x)$. If $f \in \mathcal{S R}\left(\Omega_{D},\left(\mathbb{R}_{m}\right)^{n}\right)$ such that its restriction $f_{I}$ to $D_{I}$ is a convex mapping such that $f\left(D_{I}\right) \subseteq \mathbb{C}_{I}^{n}$. If $f(0)=0, f^{\prime}(0)=\mathbb{I}_{n}$ (identity matrix of order $n$ ), then for any $x \in \Omega_{D}$,

$$
\frac{\rho(x)}{1+\rho(x)} \leq\|f(x)\| \leq \frac{\rho(x)}{1-\rho(x)}
$$

or equivalently,

$$
\frac{\|x\|}{1+\rho(x)} \leq\|f(x)\| \leq \frac{\|x\|}{1-\rho(x)} .
$$

These estimates are sharp.

\section{FinAL REMARKS}

In this paper, we have proved the growth theorem for slice monogenic extensions of starlike and convex mappings on the unit ball in the subset of slice several Clifford variables $\left(\mathcal{Q}_{m}\right)_{s}^{n}$. However, the corresponding distortion theorem is still untouched. This deserves further investigation. 


\section{REFERENCES}

[1] W. W. Adams, C. A. Berenstein, P. Loustaunau, I. Sabadini, and D. C Struppa. Regular functions of several quaternionic variables and the Cauchy-Fueter complex. J. Geom. Anal., 9(1):1-15, 1999.

[2] F. Colombo and I Sabadini. A structure formula for slice monogenic functions and some of its consequences. In Hypercomplex analysis, Trends Math., pages 101-114. Birkhäuser Verlag, Basel, 2009.

[3] F. Colombo, I. Sabadini, and D. C Struppa. Slice monogenic functions. Israel J. Math., 171:385-403, 2009.

[4] F. Colombo, I. Sabadini, and D. C Struppa. An extension theorem for slice monogenic functions and some of its consequences. Israel J. Math., 177:369-389, 2010.

[5] F. Colombo, I. Sabadini, and D. C Struppa. Noncommutative functional calculus, volume 289 of Progress in Mathematics. Birkhäuser/Springer Basel AG, Basel, 2011. Theory and applications of slice hyperholomorphic functions.

[6] F. Colombo, I. Sabadini, and D. C Struppa. Algebraic properties of the module of slice regular functions in several quaternionic variables. Indiana Univ. Math. J., 61(4):1581-1602, 2012.

[7] Gentili and D.C Struppa. A new approach to Cullen-regular functions of a quaternionic variable. C. R. Math. Acad. Sci. Paris, 342(10):741-744, 2006.

[8] Gentili and D.C Struppa. A new theory of regular functions of a quaternionic variable. Adv. Math., 216(1):279-301, 2007.

[9] Gentili and D.C Struppa. Regular functions on a Clifford algebra. Complex Var. Elliptic Equ., 53(5):475-483, 2008.

[10] Gentili and D.C Struppa. Regular functions on the space of Cayley numbers. Rocky Mountain J. Math., 40(1):225-241, 2010.

[11] R. Ghiloni and A Perotti. Slice regular functions on real alternative algebras. Adv. Math., 226(2):1662-1691, 2011.

[12] R. Ghiloni, A. Perotti, and C Stoppato. The algebra of slice functions. Trans. Amer. Math. Soc., 369(7):4725-4762, 2017.

[13] R. Ghiloni, A. Perotti, and C Stoppato. Sigularities of slice regular functions over real alernative *-algebras. Adv.Math., 305:1085-1103, 2017.

[14] R Ghiloni and Alessandro Perotti. Slice regular functions of several clifford variables. AIP Conference Proceedings, 1493:734-738, 112012.

[15] Graham I and Kohr G. Geometric function theory in one and higher dimensions. Chapman and Hall/CRC Pure and Applied Mathematics. New York : Marcel Dekker, 2003.

[16] Taishun Liu and Guangbin Ren. The growth theorem for starlike mappings on bounded starlike circular domains. Chinese Ann. Math. Ser. B, 19(4):401-408, 1998. A Chinese summary appears in Chinese Ann. Math. Ser. A 19 (1998), no. 5, 657.

[17] Taishun Liu and Guangbin Ren. Growth theorem of convex mappings on bounded convex circular domains. Sci. China Ser. A, 41(2):123-130, 1998.

[18] Guangbin Ren and Xieping Wang. Growth and distortion theorems for slice monogenic functions. Pacific J. Math., 290(1):169-198, 2017.

[19] Guangbin Ren, Xieping Wang, and Zhenghua Xu. Slice regular functions on regular quadratic cones of real alternative algebras. In Modern trends in hypercomplex analysis, Trends Math., pages 227-245. Birkhäuser/Springer, Cham, 2016.

[20] G. K. H Sprig. Holomorphic functions in the plane and n-dimensional space. Birkhuser Verlag, Basel, 2008.

[21] Haiyan Wang and Guangbin Ren. Octonion analysis of several variables. Commun. Math. Stat., 2(2):163-185, 2014.

E-mail address, G. Ren: rengb@ustc.edu.cn

E-mail address, T. Yang: @mail.ustc.edu.cn 